\title{
Intermédialités
}

Histoire et théorie des arts, des lettres et des techniques

Intermediality

History and Theory of the Arts, Literature and Technologies

\section{Bâtir. La ville intermédiale}

\section{James Cisneros et Will Straw}

Numéro 14, automne 2009

bâtir

build

URI : https://id.erudit.org/iderudit/044406ar

DOI : https://doi.org/10.7202/044406ar

Aller au sommaire du numéro

Éditeur(s)

Revue Intermédialités (Presses de l’Université de Montréal)

ISSN

1705-8546 (imprimé)

1920-3136 (numérique)

Découvrir la revue

Citer ce document

Cisneros, J. \& Straw, W. (2009). Bâtir. La ville intermédiale. Intermédialités /

Intermediality, (14), 11-18. https://doi.org/10.7202/044406ar d'utilisation que vous pouvez consulter en ligne.

https://apropos.erudit.org/fr/usagers/politique-dutilisation/ 


\title{
Bâtir. La ville intermédiale
}

\author{
James Cisneros et Will Straw
}

\section{B}

âtir renvoie historiquement à une action déterminée par un plan systématique. Cette action englobe la réalisation d'édifices ou d'espaces urbains et les diverses manières dont ils sont habités. La construction contemporaine nous montre à quel point les technologies liées aux nouveaux médias ainsi que leurs doubles réseaux, matériels et immatériels, sont au cœur de l'architecture et de la planification urbaines. Cependant, bâtir implique généralement un débordement des limites médiatiques dans la mesure où il y a toujours une transition entre deux formes de signification, à savoir un transfert du projet sur papier vers la structure architecturale. Aussi mesurable soit-elle, cette transition repose en outre sur de nombreux courants qui convergent en un même processus : du point de vue de l'architecte, ce sont les technologies du bâtiment, les systèmes domestiques de communication, l'environnement vernaculaire et les édifices alentour, à quoi s'ajoutent d'autres facteurs comme la législation, les désirs et le portefeuille du client; du point de vue du planificateur urbain, on pense à l'infrastructure des réseaux, aux schémas de circulation et de trafic, enfin aux traditions politiques ou culturelles qui sont denses et résistent au progrès ou l'accélèrent, imposant des rythmes, historiquement variables, au changement. Ainsi, parce qu'elle suppose une transposition de la feuille de papier vers l'édifice réalisé, l'action de bâtir implique un processus intermédial qui fait converger des pratiques et des formes culturelles disparates.

Ce numéro d'Intermédialités a pour thème l'environnement urbain et sa construction. Dans leur diversité, les textes réunis ici nous invitent à réfléchir à la manière dont les espaces urbains ont reçu leur forme et leur texture de différents médias ainsi qu'aux relations entre ces derniers et l'environnement bâti. Les articles s'inscrivent dans un ensemble de développements récents concernant deux champs: d'une part, la théorie des médias qui s'est tournée vers les idées de «matérialité » et d'infrastructure et dont le renouvellement se reconnaît de façon dispersée dans plusieurs des courants d'analyse culturelle; d'autre part, l'architecture et l'urbanisme au sein desquels le mouvement déconstructiviste 
a remis en question les conceptions traditionnelles de l'espace et du «sol » qui étaient censées conditionner l'action de bâtir. Dans ce second cas, l'appel à penser le «bâtir» signifie rendre compte d'une tradition de pensée modelée par une conception idéelle du «bâtir ». Il y a longtemps que les philosophes utilisent des métaphores spatiales pour fonder et stabiliser leurs systèmes de pensée. Cette tradition a imprégné l'architecture elle-même aussi bien que la planification urbaine. Elle est un des éléments qui interviennent dans la transition que nous avons appelée le «bâtir », une tradition que Kojin Karatani a retracée depuis sa source platonicienne ${ }^{1}$ et qui nous aide à illustrer les liens entre espace urbain et différents médias.

Dans Phèdre, Socrate accompagne son ami hors des murs de la ville, jusqu'à un platane au bord de la rivière. En chemin, il récuse l'interprétation des mythes par les lieux, parce que ce savoir le détourne de la connaissance de soi. Une fois arrivé, il rejette le discours écrit dont Phèdre lui fait la lecture parce que, à l'instar d'une peinture, celui-ci est incapable de répondre aux questions. Ces médias, pour les appeler ainsi, produisent des imitations de l'idée, de simples simulacres. Leur circuit de communication distend trop les limites spatiales à l'intérieur desquelles se logent les formes acceptables de la connaissance (de soi), celles qui émergent du dialogue oral dont seul l'espace circonscrit de la polis peut assurer l'efficacité. À l'inverse, les textes de Lysias, ou le poème récité par le rhapsode, doivent être chassés hors de ses murs. Une distinction épistémologique est ainsi opérée qui coïncide avec un centre urbain, un ordre politique, un médium privilégié et une subjectivité d’un genre particulier. La hiérarchie entre les médias, même si la priorité de certains médias sur d'autres pose problème², suppose donc qu'on distingue le dehors du dedans, le vrai du faux, le logos original du simulacre. En se concentrant sur des médias disparates, qui convergent ou se heurtent ${ }^{3}$, on fait interagir un ensemble d'éléments, contournant ainsi la conception traditionnelle d'une ville idéalisée, sans arêtes.

1. Voir Kojin Karatani, Architecture as Metaphor. Language, Number, Money, Cambridge (Mass.), MIT Press, 1995, qui analyse la manière dont la «volonté d'architecture » issue de Platon influence des philosophes et des penseurs du $19^{\mathrm{e}}$ et du $20^{\mathrm{e}}$ siècle, ainsi que des urbanistes comme Jane Jacobs et Christopher Alexander.

2. Dans «La pharmacie de Platon », Jacques Derrida explore l'écriture de la bivalence nécessaire à la supposée priorité de la parole. Son texte est publié dans Platon, Phèdre, Paris, Flammarion, coll. «Garnier-Flammarion», 1992, p. 255-403.

3. Henry Jenkins, Convergence Culture: Where Old and New Media Collide, New York, New York University Press, 2006. 
On peut soutenir que c'est à l'orée du $20^{\mathrm{e}}$ siècle que cette tradition a le plus fortement touché les pratiques du bâtiment, quand le modernisme a rationalisé la forme en s'inspirant des lignes (géométriques) de l'idéalisme fonctionnaliste 4 . À l'image d'autres conceptions de l'espace urbain qui ont émergé dans les années 1920 et 1930, cette histoire continue d'influer sur les pratiques du bâtiment contemporaines. Plusieurs des articles de ce numéro reviennent sur le modernisme et mettent au jour l'effet qu'il continue d'exercer sur la façon de percevoir des subjectivités politiques de la ville en mutation (Altarelli, Kinik, Read). L'architecte moderniste est un cas exemplaire d'une tendance idéaliste qui se sépare du «sol» matériel - le «sol», un mot qui, à l'instar de «fondations », informe le discours des architectes autant que celui des philosophes. La ville radieuse de Le Corbusier ${ }^{5}$ abandonne le sol naturel au profit de sites artificiels. Comme l'a montré John Rajchman, Le Corbusier prend parti pour des plateformes abstraites et des pilotis qui surplombent le sol, de sorte que son architecture se libère, aussi bien visuellement que conceptuellement, du poids des formes passées, de l'environnement vernaculaire et du voisinage ${ }^{6}$.

En vue de repenser la relation entre le sol et la structure et de remettre en question la tradition qui la sous-tend, Rajchman a réévalué la mémoire urbaine de façon à mettre en perspective les réactions directes ou implicites au projet moderniste. Les coupes architecturales de Gordon Matta-Clarke, par exemple, donnent une vue anti-monumentaliste des bâtiments qu'il dissèque. Elles en

4. Pour la dette de Le Corbusier vis-à-vis de l'idéalisme du $19^{\mathrm{e}}$ siècle, voir Ignasi de Solà-Morales, Diferencias. Topografía de la arquitectura contemporánea, Barcelone, Gustavo Gili, 1998, p. 58.

5. Le Corbusier, La ville radieuse: éléments d'une doctrine d'urbanisme pour l'équipement de la civilisation machiniste. Paris, Genève, Rio De Janeiro, Sao Paolo, Montevideo, Buenos Aires, Alger, Moscou, Anvers, Barcelone, Stockholm, Nemours, Piacé, Boulogne (Seine), Éditions de l'Architecture d'aujourd'hui, 1935.

6. John Rajchman, Constructions, Cambridge (Mass.), MIT Press, 1998, p. 77-90. Rajchman découvre une source capitale pour le cadre conceptuel que se partagent philosophie et architecture : c'est «le mot ratio donné en traduction par Grund, même si le logos, que Grund traduit par ratio, implique déjà un langage géospatial » (ibid., p. 124, note 1). Il cite la «dématérialisation» prônée par Mies van der Rohe comme un autre exemple de ce déracinement par rapport à la tradition et à la ville; le Guggenheim de Frank Lloyd Wright est encore un exemple de séparation entre l'architecture et la texture urbaine. On trouvera une discussion nuancée du modernisme et de sa relation à la tradition (particulièrement à travers une critique des approches contemporaines de Mies) dans Ignasi de Solà-Morales, «Mies van der Rohe y el minimalismo », Diferencias. Topografía de la arquitectura contemporánea, 1998, p. 27-40. 
restaurent la matérialité tout en retrouvant la mémoire sociale cachée derrière les façades. Les «cités d'excavation artificielle» de Peter Eisenman offrent un autre exemple de conception de l'espace urbain constitué de strates superposées retenant la mémoire partiellement visible de la ville, une mémoire dont Rajchman compare les connexions inégales au montage cinématographique ${ }^{7}$. Un troisième exemple pourrait être celui de Paul Virilio, qui explore les perceptions corporelles et les variations au sein de la culture de la vitesse. Il pose la question des espaces urbains propres à libérer la «trajectivité » des corps des formes centralisées de la subjectivité (politique) ${ }^{8}$. Le déplacement que subit le concept de «sol», introduit dans l'architecture par la philosophie et toujours rattaché au logos, ouvre de nouvelles possibilités à travers lesquelles on peut repenser le «bâtir». À la place des notions de structure et d'idée, de solidité et de firmitas, surgissent celles de matérialité et de mémoire, de mouvement et de sensation.

La recherche contemporaine essaie de préciser jusqu'à quel degré mouvement et circulation constituent la ville, modulant sa perception; pour cela elle puise à de nombreuses sources des années 1920 et 1930 en particulier. Dans le droit fil d'une réflexion entamée par Georg Simmel, Walter Benjamin et Siegfried Kracauer abordent la métropole dans l'optique des pratiques émergeant de la quotidienneté, pratiques qui en sont venues à définir le dynamisme de la vie urbaine. Cette vision de la modernité, très éloignée de celle impliquée par le modernisme architectural, reste valide quand il s'agit d'étudier les villes d'aujourd'hui. Plusieurs des articles de ce numéro explorent la manière dont les trajectoires corporelles s'ouvrent sur des perceptions de l'environnement bâti marquées de nouveauté et «d'estrangement» (Perivolaropoulou, Robin, Thain). D'autres s'attachent à des questions voisines surgies à la même époque - chez Paul Valéry entre autres - et dont l'objet est de s'interroger sur la façon dont les technologies de l'archive et de la transmission modifient l'interface sensorielle avec l'espace urbain (Darò, Altarelli).

Si notre approche du bâtir comme processus intermédial emprunte ces questionnements à l'architecture et à l'urbanisme, elle rejoint aussi les récents développements de la théorie des médias. Dans les deux cas, c'est la matrice d'événements inhérente à une conception idéelle du «bâtir» qui se voit

7. Voir également Jean-François Bédard (dir.), Cities of Artificial Excavation: The Work of Peter Eisenman, 1978-1988, Montréal, CCA, 1994.

8. Rajchman renvoie ses lecteurs à Paul Virilio, Cybermonde, la politique du pire: entretiens avec Philippe Petit, Paris, Textuel, 1996, 23 et sq. 
réévaluée. Emboîtant le pas à Valéry, Friedrich Kittler s'est demandé si «la ville est un médium ${ }^{9}$ » La réponse (non conclusive) qu'il élabore nous incite à considérer plus largement les villes en relation avec leur médialité. Par médialité, nous désignons les manières dont les artefacts culturels (œuvres relevant des beauxarts ou de la culture populaire, ornements apportés à l'infrastructure urbaine, etc.) participent de ce que Kittler décrit comme la capacité « d'enregistrer, transmettre et traiter l'information ${ }^{10} »$. Il s'interroge ainsi sur la faculté de la ville à devenir, en tant que médium, un moyen de contrôler la circulation des biens et de l'information, de limiter l'accès à l'espace, de canaliser la population et les débats politiques. La médialité, en ce sens, est une propriété de tous les artefacts culturels (par exemple les plaques apposées sur les murs comme supports de textes qui peuvent être poétiques ou historiques). Il ne s'agit pas de désigner simplement par là ces technologies, telles la télévision ou Internet, que par convention on appelle «médias». Les formes médiales stockent et transmettent l'expression culturelle selon des modalités propres; elles traceraient donc les contours qui contiennent cette expression et la modèlent.

D’autres théories des médias qui mettaient l'accent sur les idées de «matérialité» et d'«extériorité» ont ouvert de nouvelles possibilités pour comprendre l'environnement bâti. Le mot « extériorité» ne désigne pas seulement ces dimensions des médias que seraient le hardware ou l'emballage. Le terme nous invite à prendre en compte l'immersion dans le matériel qui caractérise l'expression culturelle, de voir comment celle-ci s'inscrit (par l'écriture) ou se répète (par la performance) au sein d'ensembles technologiques, dans les corps et les structures physiques. Les récentes recherches anthropologiques de Gaonkar et de Povinelli, dont nous nous inspirons, s'adressent aux chercheurs en études culturelles pour les inciter à analyser les «contours des formes» telles qu'elles circulent. Ils soutiennent, comme certains articles de cette livraison (Froger, Read), que cette

9. Il s'inspire de «La conquête de l'ubiquité», qui est aussi le point de départ du célèbre essai de Benjamin sur l'œuvre d'art à l'époque de sa reproductibilité technique. Dans un autre texte, Valéry réagit avec esprit à la tradition platonicienne dont il a été question plus haut: Eupalinos, ou l'architecte [1923], Paris, Gallimard, coll. «NRF», 1944, qui a été publié la même année que Vers une architecture de Le Corbusier, Paris, Éditions Georges Crès et Cie, 1923. Friedrich A. Kittler, «The City is a Medium », New Literary History, vol. 27, $\mathrm{n}^{\circ}$ 4, automne 1996, p. 717-729; la citation se trouve p. 722 .

10. Ibid. 
circulation est fondamentale quand il s'agit de donner de la texture à la vie sociale et à ses expressions politiques ${ }^{11}$.

Les incitations à étudier les «matérialités» de la communication et ses «arêtes formelles» représentent d'importantes incursions dans la théorie des médias qui nous poussent également à réviser notre manière de penser les villes. La ville est constituée de manière significative par ces matérialités et ces «arêtes formelles». Les trajets suivis par les employés de la poste ne nous disent rien sur le contenu des lettres et des paquets; cependant, dans la mesure où les villes sont organisées comme des systèmes de trajets et d'adresses, il existe une rationalité spatiale qui se construit sur la circulation de l'expression intime ${ }^{12}$. Il se produit presque inévitablement une médiatisation des arêtes physiques que présente l'environnement urbain, soit en raison des fonctions que ces arêtes exercent en tant que repères, soit parce qu'elles deviennent littéralement des supports pour des inscriptions ou des textes. L'information ou l'expression culturelle ne traversent pas simplement la ville comme un nuage, elles deviennent prétexte à bâtir des structures, organiser l'espace, poser des interfaces (comme le téléphone public) dans certains lieux et à l'intention de gens assemblés autour de bornes médiatiques (comme le poste de télévision au café des sports) ${ }^{13}$. Selon l'angle d'approche, chacune des théories qui sont reconsidérées dans ce volume renverse une tradition précise; il s'agit en effet d'analyser la manière dont les fonctions exercées par les médias constituent véritablement la ville, plutôt que la manière dont la délimitation d'une zone urbaine détermine des clivages spatiaux, la hiérarchie entre les médias, les formes de subjectivité (politique) ou encore la possibilité de penser l'environnement bâti.

Les articles rassemblés dans ce volume offrent une riche variété d'approches consacrées aux relations entre architecture, ville et médias. Ils s'appliquent à une nouvelle réflexion sur l'action de «bâtir» en privilégiant trois axes qui se croisent, et dont on trouve des échos d'intensité diverse dans chacune des études: l'estrangement ressenti face au paysage urbain familier; les effets des nouvelles technologies sur les pratiques du bâtiment; enfin, la reformulation des liens et des clivages caractérisant l'espace politico-social.

11. Dilip Parameshwar Gaonkar et Elizabeth A. Povinelli, «Technologies of Public Forms: Circulation, Transfiguration, Recognition", dans Public Culture, vol. ${ }_{15}, \mathrm{n}^{\circ} 3$, 2003, p. $385-398$.

12. Voir par exemple Bernhard Siegert, Relays: Literature as an Epoch of the Postal System, Stanford, Stanford University Press, 1999.

13. Anna McCarthy, Ambient Television: Visual Culture and Public Space, Durham, Duke University Press, 2001. 
Par des voies très différentes, les textes de Nia Perivolaropoulou, d'Alanna Thain et de Régine Robin traitent de la sensation d'estrangement que les espaces familiers produisent dans les villes ainsi que du statut médial non conventionnel de ces espaces. S'attachant de près à une série de scénarios rédigés par Siegfried Kracauer dans les années 1930, Perivolaropoulou s'intéresse à la façon dont ils proposent de filmer l'étrangeté de la ville avec des images et un son déformants pour l'imaginer du point de vue d'un touriste étranger ou d'un jeune enfant. Elle porte ici un regard neuf, d'autant plus qu'elle fait dialoguer ces scripts avec les célèbres essais de Weimar que Kracauer a consacrés à la culture urbaine et aux masses salariées, voire avec sa Theory of Film parue ultérieurement. Dans les analyses de Thain, l'ascenseur est un médium qui transporte et relie, mais qui peut tout aussi bien devenir un espace d'horreur quand il échappe aux systèmes de surveillance informels qui garantissent la sécurité dans la vie urbaine. Par contraste, la face médiale du Tokyo de Régine Robin est tournée vers le dehors, et la surface de la ville devient alors presque intégralement une prolifération de réseaux, de câbles et de signes offrant autant de formes de lisibilité qu'il y a de trajectoires dans les rues.

Un second groupe de textes explore les nouvelles pratiques du bâtiment rendues possibles par les innovations technologiques et montre comment cellesci modifient le paysage urbain sur un plan à la fois physique et imaginaire. Lucio Altarelli étudie le passage de la lumière incandescente caractéristique de l'architecture moderne à la lumière électronique de l'architecture contemporaine: il analyse la manière dont se déploient ainsi des paysages urbains et des pratiques culturelles spécifiques. Cette concentration sur l'éclairage artificiel lui permet d'explorer l'histoire de cette architecture qui s'est éloignée du modernisme de Le Corbusier et du Style international pour s'engager dans les formes d'expression propres à la culture digitale. Carlotta Darò s'attache de son côté à la discothèque, un espace qui a souvent été imaginé sous l'angle de l'utopie et de la fête, avec ses éléments médiaux tournés vers le dedans produisant un monde circonscrit et sensoriellement plein. Elle montre comment les nouveaux développements technologiques ont fait de la «boîte de nuit » une sorte de laboratoire des pratiques culturelles urbaines pour les groupes italiens néo-avant-gardistes des années 1960 et 1970 - Archizoom et Superstudio -, qui élaboraient alors une réflexion nouvelle sur la fonction et le style en recherchant des formes inédites d'inspiration idéologique.

Finalement, un groupe de textes met en perspective la ville en tant que polis, en tant qu'espace social zébré de technologies médiatiques et de formes de représentation multiples. Anthony Kinik examine de récents films, tournés 
par le réalisateur Patrick Keiller, ayant Londres pour sujet. Il les resitue au sein d'une longue tradition, celle de la «city symphony» qui a cherché à développer des homologies entre les formes cinématiques et celles des villes elles-mêmes. Dans la lignée des analyses politiques des mutations urbaines (Lefebvre, Harvey), il compare les écrits de Keiller sur l'évolution de l'espace moderniste au foyer critique de ces films consacrés aux «new spaces» qui ont surgi en Angleterre avec l'économie néo-libérale. Justin Read, quant à lui, étudie les représentations de Rio de Janeiro dans la poésie et l'architecture modernistes ainsi que dans la télévision postmoderne. Il propose une analyse fortement politique de la manière dont ces divers moyens d'expression culturelle sont reliés à des formes de pouvoir institutionnelles. Reprenant la distinction opérée par Lefebvre entre espace abstrait et espace absolu, il montre les tensions et les contradictions qui marquent la tentative de l'État-nation d'intégrer les citoyens par des formes médiatisées qui continuent de résister à sa représentation cartographique. La rue ainsi que les projections chevauchant l'espace urbain subissent un déplacement historique sous l'effet des nouvelles technologies et des nouvelles formes de pouvoir. L'article de Marion Froger, enfin, traite d'un sujet longtemps considéré comme majeur dans les études de communication urbaine: il s'agit du rôle des médias dans la production du lien social qu'elle étudie en faisant appel à la notion de mitoyenneté, un néologisme inventé par Isaac Joseph pour contourner les connotations historiquement chargées du terme de citoyenneté. Froger prend pour objet l'œuvre du réalisateur de documentaires néerlandais Johan van der Keuken. Elle y repère de multiples stratégies pour élaborer des relations de réciprocité entre réalisateur et sujet, qu'il s'agisse du travelling qui maintient temporellement la relation dans la durée, ou encore de la manière dont la caméra recherche consciemment le consentement de ceux qui sont filmés.

Traduit de l'anglais par Caroline Bem 